\title{
Estimador de Coeficientes de Canal DS/CDMA Baseado em Filtragem de Partículas
}

\section{Particle Filtering for Multiple Access DS/CDMA Systems DS/CDMA Channel Estimation}

\author{
Rafael O. Ribeiro' ${ }^{1}$; Taufik Abrão
}

\begin{abstract}
Resumo
Este artigo discute aspectos de implementação computacional e desempenho da metodologia Bayesiana Filtro de Partículas. Esta técnica de estimação é diretamente aplicada à estimativa dos coeficientes de canal em um sistema DS/CDMA. Os resultados da simulação para um canal de propagação Rayleigh sem linha de visada indicaram que o estimador Bootstrap PF é capaz de fornecer RMSE no intervalo de $\left[10^{-3} ; 10^{-2}\right]$ para uma grande faixa de nível de interferência de múltiplo acesso (MAI) e relação sinalruído (SNR), e ainda ser capaz de oferecer robustez para o efeito near-far.

Palavras-chave: Estimação de parâmetros de canal. Estimador Bayesiano. Filtro de partículas. DS/ CDMA.
\end{abstract}

\begin{abstract}
This article discusses computational implementation aspects and performance of a Bayesian methodology, namely particle filter (PF). The PF channel estimation technique is directly applied to the channel coefficients estimation of DS/CDMA systems. Simulation results for non-line-of-sight (NLOS) Rayleigh fading channel propagation have indicated that the bootstrap PF estimator is capable to provide RMSE in the range of $\left[10^{-3} ; 10^{-2}\right]$ for a wide range of multiple access interference (MAI) levels and signal-noise ratio (SNR), and still be able to offer robustness to near-far ratio (NFR) effect. Keywords: Channel parameters estimation. Bayesian estimator. Particle filter. Bootstrap resampling. DS/CDMA.
\end{abstract}

\section{Introdução}

A busca por maior mobilidade aliado às características de altas taxas de transmissão, fizeram com que novas tecnologias de estimação/recepção fossem desenvolvidas visando a otimização dos sistemas de comunicações.
Muitos parâmetros de interesse não podem ser medidos/estimados de forma perfeita, uma vez que tais medidas normalmente são corrompidas por ruído e em muitos cenários por interferência de múltiplo acesso. Portanto, faz-se necessária a aplicação de ferramentas/técnicas estatísticas na

1 R. O. Ribeiro é Engenheiro de Proteção do Sistema de Distribuição da COPEL e mestrando do Programa de Pós-Graduação em Engenharia Elétrica da Universidade Estadual de Londrina (UEL), Londrina, Paraná, Brasil, rafael.rib87@gmail.com rafael. oribeiro@copel.com

2 T. Abrão é Professor Associado da Universidade Estadual de Londrina (UEL), Londrina, Paraná, Brasil, taufik@uel.br abrao@ ieee.org

Semina: Ciências Exatas e Tecnológicas, Londrina, v. 34, n. 1, p. 107-116, jan./jul. 2013 
obtenção de estimativas das variáveis observadas com menor erro possível.

O filtro de partículas (PF - Particle Filter) é um método estatístico Bayesiano constituído de técnicas probabilísticas recursivas aplicáveis à estimação e atualização de estados dinâmicos de um sistema a partir de uma base parcial de conhecimento do processo.

No campo de aplicação de interesse, i.e., em sistemas de comunicação de múltiplo acesso por espalhamento espectral de sequência direta (DS/ CDMA) de grande capacidade e desempenho, uni- ou multi-portadoras, faz-se necessário obter parâmetros de canal com relativa precisão, conjugada a uma complexidade moderada. Em Hu et al. (2007) é apresentado um receptor para sistemas CDMA que realiza estimativa dos coeficientes de canal e dos símbolos transmitidos baseada na metodologia Bayesiana VB-SAGE (variational Bayesian spacealternating generalized expectation-maximization), onde a estimativa para a distribuição de probabilidade dos coeficientes de canal é combinada à detecção da sequência de bits transmitidos e atualizados de forma iterativa e alternada.

Em Ciriaco et al. (2009) é proposta uma metodologia baseada na meta-heurística Algoritmo Genético (GA - Genetic Algorithm) para estimação do módulo e fase dos parâmetros do canal, utilizando a regra de máxima verossimilhança (ML - Maximum Likelihood) para encontrar o vetor solução que maximiza a função resposta do canal de acordo com os bits transmitidos e das sequências de espalhamento de todos os usuários, enquanto em Guimarães (2005) os autores aplicam a técnica de decomposição em subespaços como base para a determinação destes parâmetros. Já em Fu e Jia (2010) é implementada uma nova metodologia de reamostragem aplicada ao filtro de partículas, visando reduzir o empobrecimento das amostras e manter a diversidade das partículas e consequentemente melhorar o desempenho do algoritmo. Em Han e Liang (2009) é proposto um novo algoritmo de filtragem por partículas para a deteç̧ão multiusuário (MUD) em sistemas CDMA com canal Rayleigh, porém não é realizado nenhum estudo sobre a estimação dos coeficientes de canal para tal sistema.

Este trabalho apresenta uma análise e caracterização de um estimador de coeficientes de canal móvel DS/CDMA, baseado no método Bayesiano por filtragem de partículas bootstrap e reamostragem do tipo Multinomial. Na Seção II é apresentado o método Bayesiano de estimação da função densidade de probabilidade (pdf) dos coeficientes do canal por filtragem de partículas. Já na Seção III é feita uma breve revisão sobre o modelo de canal (transmissão/recepção) DS/CDMA utilizado no trabalho. Na Seção V são discutidos os resultados numéricos obtidos para estimação dos coeficientes de canal Rayleigh em sistemas DS/ CDMA. Por fim, na Seção VI são apresentadas as conclusões deste trabalho.

\section{Filtro de Partículas}

A filtragem por partículas é uma técnica sequencial de Monte Carlo baseada na metodologia estatística Bayesiana, a qual realiza cálculos das distribuições de probabilidades relevantes utilizando os conceitos de amostragem. Essas distribuições são aproximadas por medidas aleatórias discretas denominadas partículas, as quais são atribuídos pesos específicos (DJURIC et al., 2003).

A reconstrução da $p d f$ do processo a ser estimado garante à técnica $\mathrm{PF}$ grande potencial de desempenho na estimação de parâmetros. Porém, um dos problemas enfrentados é a degeneração dos pesos das partículas que ocorre devido à variabilidade crescente destes pesos ao longo das iterações, fazendo com que se tenha um grande esforço computacional para sua atualização.

Assim, faz-se necessário técnicas de reamostragem para evitar tal problema, já que 
com a reamostragem, as partículas de baixa probabilidade (pequenos pesos) são removidas e as de alta probabilidade (pesos grandes) são mantidas e replicadas, diminuindo a degeneração das partículas. Entretanto, com a reamostragem pode-se ter uma perda na diversidade das partículas causada pela replicação das de maior peso, já que as trajetórias simuladas das partículas não são estatisticamente independentes.

Para este trabalho foi utilizado a técnica bootstrap particle filter, BPF, conjugado ao método de reamostragem multinomial, uma vez que a BPF multinomial apresenta baixa complexidade computacional, atingindo o mesmo desempenho de outras técnicas de filtragem por partículas. Uma análise de desempenho das diferentes técnicas de PF combinadas a distintos métodos de reamostragem é apresentada em Ribeiro, Mussi e Abrão (2011).

O método bootstrap (GORDON; SALMOND; SMITH, 1993) é uma das primeiras implementações práticas de filtragem, sendo uma opção viável quando comparada aos outros métodos. Esta técnica de baixa complexidade baseia-se na ideia da amostragem sequencial por importância combinada à reamostragem, pois apenas a amostragem sequencial por importância acarretaria em uma degeneração das partículas, tornando impreciso o processo de estimação.

\section{Modelo de Sistema}

O cenário proposto consiste de um sistema DS/ CDMA com modulação BPSK. O modelo de canal utilizado considera que os coeficientes de canal são modelados como um processo aleatório de Rayleigh para módulo e Uniforme para a fase. Para a recuperação da informação no receptor de forma coerente, faz-se necessária a estimação dos parâmetros de canal. Assim, devem-se inserir periodicamente símbolos pilotos na transmissão para verificar como o canal se comporta e consequentemente estimar o mais precisamente possível os parâmetros associados ao canal. Essa inserção de símbolos pilotos acarreta em overhead na transmissão, podendo reduzir a eficiência do sistema, pois parte da energia e tempo da transmissão estão sendo dedicados ao envio de símbolos pilotos e não de informação propriamente dita Haykin e Moher (2008). Assim, o PF irá atuar na etapa de modo de treinamento supervisionado (supervised training mode), onde o receptor conhece a informação enviada (sequência de curta duração) e realiza uma estimativa do canal.

\section{Sistemas DS/CDMA}

A modulação por espalhamento espectral utilizando sequência direta (DS) faz com que o sinal transmitido seja espalhado por uma faixa de frequência mais ampla do que a largura de banda mínima necessária para transmissão: uma sequência de dados de entrada multiplicada no domínio do tempo por um código de espalhamento, cujo período de chip é muito menor que o período de bit de dados, $\mathrm{T}_{\mathrm{c}}<<\mathrm{T}_{\mathrm{b}}$. Assim, transforma-se a sequência de dados de banda estreita em um sinal de banda larga, cujo espectro torna-se semelhante ao do ruído térmico.

O sistema DS/CDMA uniportadora utiliza uma sequência distinta para identificar cada usuário. Esse sistema caracteriza-se pela interferência de múltiplo acesso (MAI), a qual ocorre devido a não-ortogonalidade perfeita que tais códigos de espalhamento apresentam.

A detecção convencional em sistemas DS/CDMA ocorre através correlação do sinal recebido ao código de espalhamento, onde este sinal recebido é composto pela superposição espectral e temporal dos sinais transmitidos pelos diversos usuários. Porém, a adequada recepção de detecção da informação é dificultada por problemas inerentes ao canal de rádio móvel, tais como perda de percurso, sombreamento, interferência de múltiplo acesso, efeito near-far e principalmente desvanecimentos profundos.

Utilizando-se modulação BPSK, o sinal síncrono equivalente banda-base à entrada do receptor pode ser expresso por: 


$$
r(t)=\sum_{k=1}^{K} A_{k} b_{k} c_{k} s_{k}(t)+\eta(t)
$$

$y_{1}=\int_{0}^{T} r(t) s_{1}(t) d t=b_{1} \sqrt{E_{b}}+\eta_{1}+\sqrt{E_{b}} \sum_{k=2}^{K} b_{k} \rho_{k}$

sendo $K$ o número de usuários, $A_{k}$ a amplitude do sinal transmitido do $k$-ésimo usuário, $b_{\mathrm{k}} \in[ \pm 1] \mathrm{o}$ bit transmitido, admitido equiprovável; $c_{k}$ e $s_{k}$ são o coeficiente de canal e a sequência de espalhamento associados ao $k$-ésimo usuário, respectivamente; $\eta(t)$ é o ruído AWGN à entrada do Rx.

No receptor, o sinal $r(t)$ deve ser multiplicado pela sequência de espalhamento do usuário $s_{k}$ de interesse, sendo o sinal à saída do banco de filtro casados (detector convencional) determinado por Abrão, Ciriaco e Jeszensky (2004):

$y_{1}=\int_{0}^{T} r(t) s_{1}(t) d t=b_{1} \sqrt{E_{b}}+\eta_{1}+\sqrt{E_{b}} \sum_{k=2}^{K} b_{k} \rho_{k}$

sendo $\rho_{k j}$ o valor da correlação cruzada entre o $j$-ésimo interferente e o usuário de interesse, $k$. Neste trabalho, adotou-se sequência de espalhamento aleatórias (PN) Torrieri (2005), justamente para demonstrar o desempenho do estimador na condição de pior caso de operação de sistema do ponto de vista de interferência de múltiplo acesso média.

\section{Interferência de Múltiplo Acesso}

A estratégia de acesso múltiplo por divisão de código e sequência direta (DS/CDMA) baseia-se no espalhamento proporcionado pelos códigos. Caso estes códigos resultassem perfeitamente ortogonais no receptor seria possível alcançar/ atingir capacidade infinita no sistema. No entanto, por limitações físicas, esta condição não pode ser atingida em sistemas práticos.

Seja a detecção do sinal relativo ao primeiro usuário $(k=1)$, o receptor convencional aplica o filtro casado (correlacionador) à sequência de assinatura de interesse $s_{l}(t)$, obtendo: sendo $E_{b}$ a energia de bit e os primeiros dois termos equivalentes ao caso de único usuário ativo no sistema. O último termo representa a MAI.

Admitindo-se o controle de potência dos transmissores, tem-se que a potência transmitida de cada usuário é ajustada dinamicamente de forma que os sinais recebidos de todos os usuários apresentem o mesmo nível.

\section{Efeito near-far}

Em um ambiente de múltiplo acesso por divisão de código, sinais oriundos de transmissores mais próximos do receptor podem causar grande interferência sobre o sinal de interesse caso não haja mecanismos de controle de potência, permitindo assim o efeito perto-longe (NFR). Para evitar este efeito, deve-se estabelecer um rigoroso esquema de controle de potência, de tal sorte a garantir que todos os sinais sejam recebidos com a mesma potência. Neste trabalho é apresentada uma análise do comportamento do estimador Bayesiano para os coeficientes de canal em sistemas DS/CDMA sob estrito efeito near-far.

\section{Modelo de Canal}

Como já relatado anteriormente, foi adotado para simular o canal o modelo de Jakes Modificado. Inicialmente proposto por JAKES (1974) e posteriormente modificado por Dent, Bottomley e Croft, (1993), o modelo de Jakes é um método determinístico para a simulação de canal com desvanecimento Rayleigh com formas de onda descorrelacionadas no tempo.

Esse efeito sobre a envoltória do sinal recebido pode ser descrito por uma distribuição estatística caracterizada pela função densidade de probabilidade de Rayleigh 


$$
p(r)=\frac{2 r}{\sigma^{2}} e^{\frac{-r^{2}}{\sigma^{2}}}
$$

onde $r$ é a amplitude instantânea do sinal recebido e $\sigma^{2}$ é a variância de $r$.

De forma análoga, a fase do sinal recebido é adequadamente modelada por uma distribuição uniforme no intervalo $[0,2 \pi]$.

\section{Estimador de Canal Bootstrap PF com Reamostragem Multinomial}

Todas as simulações realizadas neste trabalho são baseadas no Método de Simulação Monte Carlo (MCS - Monte Carlo Simulation).

A robustez do sistema pode ser avaliada através dos efeitos de carregamento do sistema e NFR. Para as simulações do sistema CDMA em canal Rayleigh foi utilizado sequência PN com ganho de processamento $\mathrm{N}=32$.

O Algoritmo 1 apresenta o pseudocódigo de implementação do BPF com reamostragem Multinomial utilizado no estimador dos coeficientes de canal. No algoritmo é realizada a reamostragem das partículas com base nos seus pesos, caso contrário, após algumas iterações grande parte das partículas estariam com peso próximo de zero, e apenas algumas partículas próximas das regiões com alta probabilidade. Assim, realizou-se a reamostragem baseada na degeneração das partículas.

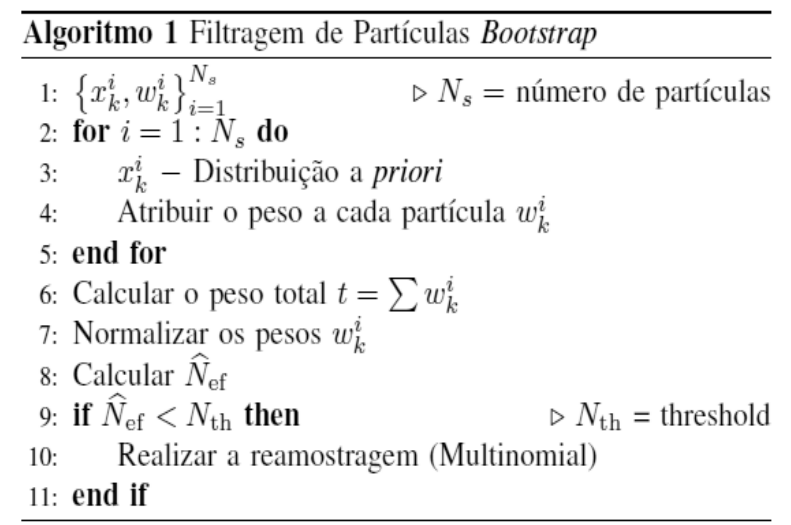

Fonte: do autor
Para determinar a degeneração das partículas, foi calculado o tamanho efetivo da amostra baseado no coeficiente de variação dos pesos. Esta estimativa é dada por:

$$
N_{e f}=\frac{1}{\sum_{i=1}^{N_{s}} w_{i}^{2}}
$$

sendo $w_{i}$ os pesos das partículas. Assim, define-se um método de rejeição comparando-se o tamanho efetivo $\left(N_{e f}\right)$ a um número de partículas de limiar $\left(N_{t h}\right)$, ou seja, quando $N_{e f}$ é inferior ao limiar, a reamostragem é realizada. Para as simulações numéricas utilizou-se o limiar de $\mathrm{N}_{\text {th }}=0,25 \mathrm{~N}_{\mathrm{s}}$ conforme encontrado na literatura, o qual garante um desempenho satisfatório para o estimador BPF.

\section{Complexidade do Estimador}

A Tabela 1 apresenta a complexidade computacional do estimador por filtragem de partículas Bootstrap com reamostragem Multinomial (M-BPF) analisado. Observa-se que tanto o número de somas como o de multiplicações depende do número de partículas, $\mathrm{N}_{\mathrm{s}}$.

Tabela 1 - Análise de complexidade do estimador BPF.

\begin{tabular}{cc}
\hline Complexidade & Estimador BPF \\
\hline Somas & $55 \mathrm{Ns}$ \\
\hline Multiplicações & $478 \mathrm{Ns}$ \\
\hline
\end{tabular}

Fonte: do autor.

De acordo com Ribeiro, Mussi e Abrão (2011) foi confirmado a menor complexidade computacional do algoritmo BPF, em termos de número de somas e multiplicações.

Outra vantagem é a manutenção do desempenho em relação às outras técnicas de filtragem de partículas minuciosamente analisadas em Ribeiro, Mussi e Abrão (2011), sendo, portanto, empregado aqui na estimação de coeficientes de canal do sistema DS/CDMA. 


\section{Número de Partículas}

A escolha do número de partículas é raramente mencionada na literatura. Uma vez que a cada iteração os pesos das partículas devem ser atualizados através da reamostragem, a determinação do número ótimo de partículas é uma tarefa fundamental para a eficiência do algoritmo (LINZHOU et al., 2010). Assim, foi realizado um estudo para determinar o número ótimo de partículas, especificamente para o problema da estimação de parâmetros de canal de rádio móvel, levando-se em conta o desempenho do estimador em termos de erro quadrático médio (MSE), bem como da complexidade computacional do algoritmo. Uma vez que estas duas figuras de mérito são dependentes do número de partículas, sob distintas condições de operação do sistema, ie., variou-se a SNR sob condição específica de carregamento de sistema $\mathrm{L}=K / N$ elevados, sendo $N=T_{b} / T_{c}$ o ganho de processamento do sistema. Assim, para carregamentos mais baixos o número de partículas ótimo encontrado também seria razoável.

A Figura 1 apresenta o desempenho (módulo e fase) do estimador de canal (MSE x Ns x SNR). Observa-se que o estimador operando com um número de partículas na faixa de [200; 400] é capaz de atingir um desempenho favorável em termos de MSE. O estimador M-BPF atinge valores de MSE $\approx 2 \times 10^{-3}$ para a estimação do módulo dos coeficientes de canal. Para valores mais elevados de números de partículas, o algoritmo tem sua complexidade computacional elevada, aumentando assim o tempo de processamento do filtro de partículas para a realização das estimações, sem, no entanto, obter melhoria no MSE compatível que justifique um aumento na complexidade.
Figura 1 - Desempenho do estimador M-BPF em função da variação da SNR e do número de partículas: a) MSE para o módulo do coeficiente de canal; b) MSE para a fase.
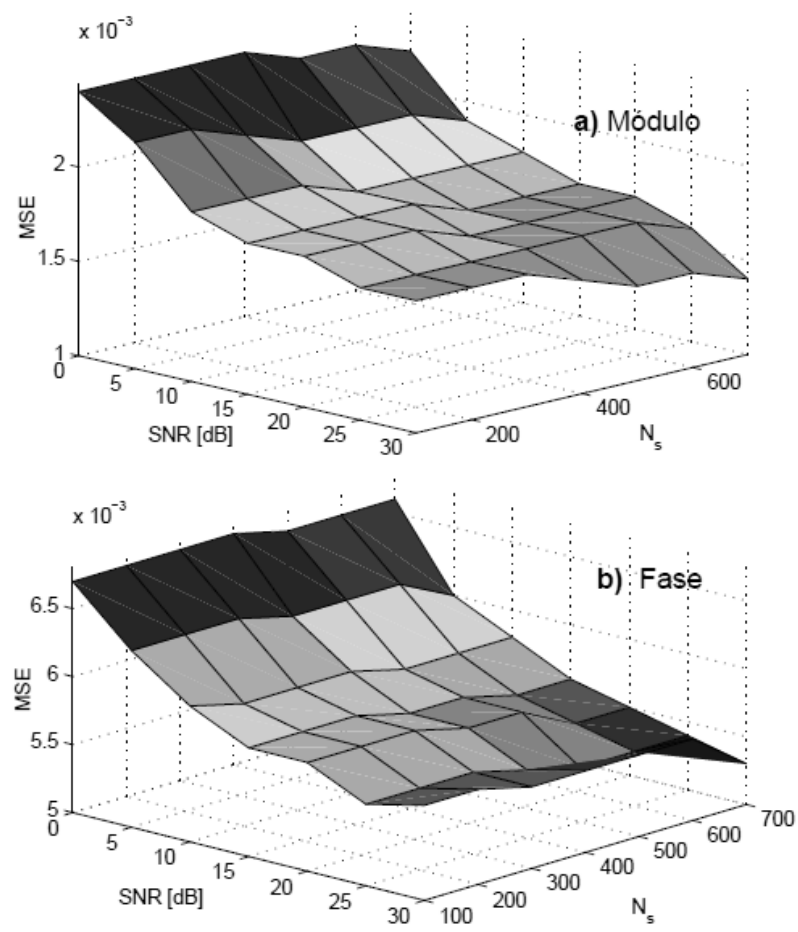

Fonte: do autor

Deve-se levar em conta que com o aumento de número de partículas $\left(\mathrm{N}_{\mathrm{s}} \rightarrow \infty\right)$, uma estimativa assintoticamente ótima da distribuição a posteriori seria obtida (objetivo da estimação Bayesiana) (CANDY, 2007). Entretanto, este aumento acarretaria em um considerável incremento da complexidade do estimador de parâmetros de canal. Assim, adotou-se $\mathrm{N}_{\mathrm{s}}=300$ partículas para o algoritmo M-BPF, sendo um número razoável, visto que esse valor garante um desempenho satisfatório com a manutenção da complexidade computacional em um patamar factível.

\section{Resultado Numérico do Estimador M-BPF}

A Figura 2 apresenta o desempenho do estimador de parâmetros de canal para um sistema DS/CDMA uniusuário, i.e., na ausência do efeito da MAI. 
Pôde-se observar que o estimador do módulo dos coeficientes de canal apresentou um desempenho superior ao da estimação da fase, principalmente para a região de alta SNR, na qual a estimação do módulo teve um desempenho melhor de quase uma década em relação à fase.

Figura 2 - Desempenho do estimador sem efeito da MAI ( $\mathrm{K}=1$ usuário).

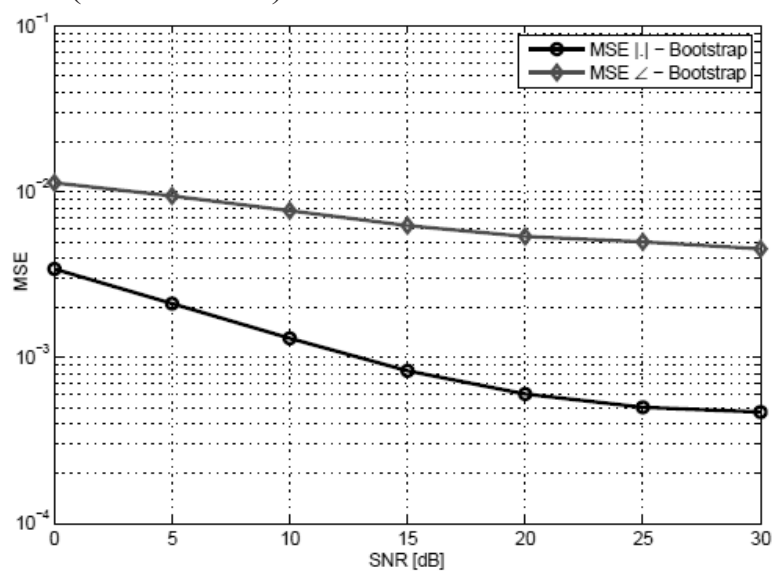

Fonte: do autor.

Figura 3 - Degradação de desempenho do estimador M-BPF com o aumento da MAI.

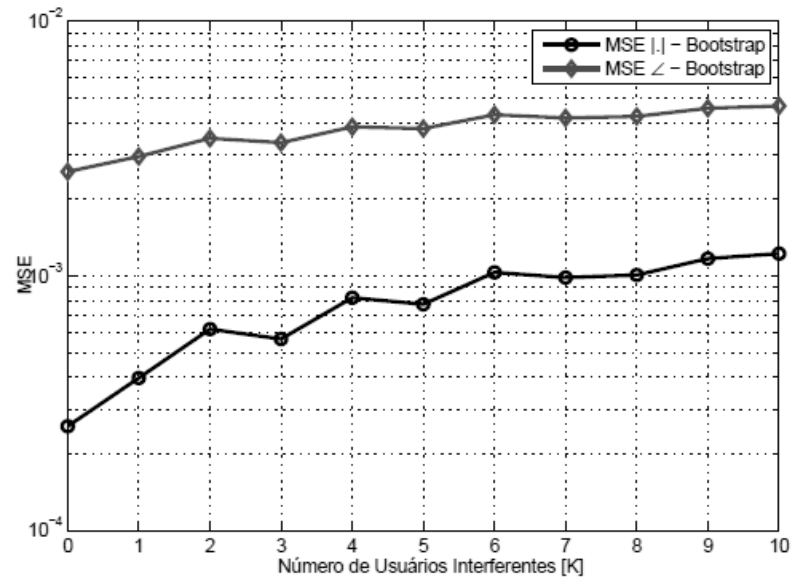

Fonte: do autor.

A Figura 3 mostra os resultados obtidos para o desempenho do estimador em relação ao aumento da MAI. Foi estabelecida uma SNR $=30 \mathrm{~dB}$ para esta simulação, ganho de processamento $\mathrm{N}=32$ e aumentou-se o índice de carregamento para verificar o comportamento do estimador em relação a interferência de múltiplo acesso. Verificou-se que o estimador não é totalmente imune ao efeito da MAI, tendo seu desempenho degradado com o aumento do carregamento do sistema. No entanto, mesmo para um carregamento de $\mathrm{L}=0,344$, o estimador mostrou bom desempenho em termos de MSE, resultando em $\mathrm{MSE}_{\mathrm{mod}} \approx 1,2 \times 10^{-3} \mathrm{e} \mathrm{MSE}_{\mathrm{ph}}$ $\approx 5 \times 10^{-3}$.

Na Figura 4 são apresentados os resultados do desempenho do Particle Filter em relação ao efeito near-far, onde foi estabelecida uma SNR $=10 \mathrm{~dB}$ para o usuário de interesse, variando-se a SNR dos interferentes. Para esta simulação foi definido 1 usuário de interesse e 3 interferentes. Verificouse que o desempenho do estimador M-BPF para o usuário de interesse é levemente reduzido em termos da estimação do módulo e fase à medida que o efeito NFR cresce. Porém, a diferença de desempenho MSE entre a estimação do módulo e fase segue a mesma tendência das figuras anteriores, uma diferença de $1 / 2$ década para toda a faixa de $\mathrm{NFR} \in[-10 ; 10] \mathrm{dB}$.

Figura 4 - Desempenho do estimador M-BPF para o efeito near-far, $\mathrm{K}=4$ usuários e $\mathrm{SNR}=10 \mathrm{~dB}$.

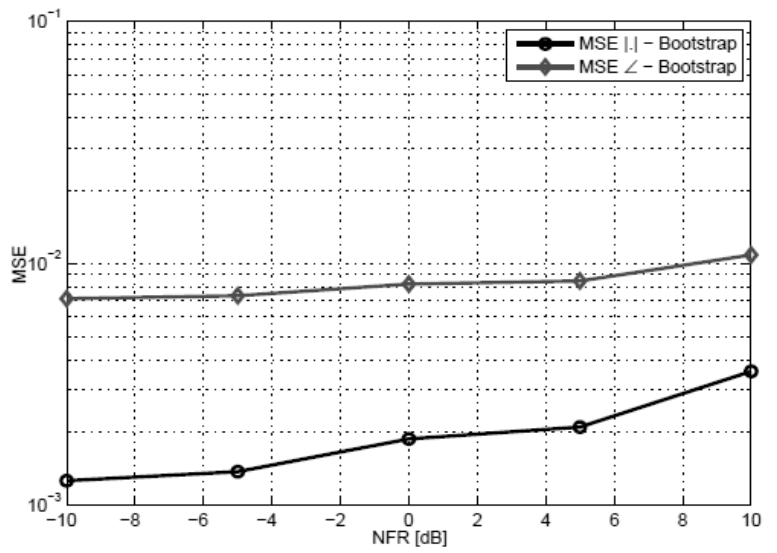

Fonte: do autor.

Finalmente, a Figura 5 apresenta os resultados do desempenho do estimador em termos do erro quadrático médio para as estimativas dos coeficientes de canal, módulo e fase. A degradação de desempenho MSE é obtida em função do aumento 
do índice de carregamento $\mathrm{L}=\mathrm{K} / \mathrm{N}$ e da redução da SNR. Novamente, observa-se que mesmo com a tendência de degradação, para a condição de pior caso analisado, i.e., faixa de carregamento de sistema de $\approx 40 \%$ e SNR $=0 \mathrm{~dB}$ o desempenho do estimador ainda é apreciável: $\mathrm{MSE}_{\text {mod }} \approx 2,2 \times 10^{-3} \mathrm{e}$ $\mathrm{MSE}_{\mathrm{ph}} \approx 6,5 \times 10^{-3}$.

Figura 5 - Desempenho do estimador M-BPF em função do incremento da SNR e da MAI: a) MSE para o módulo; b) MSE para a fase.
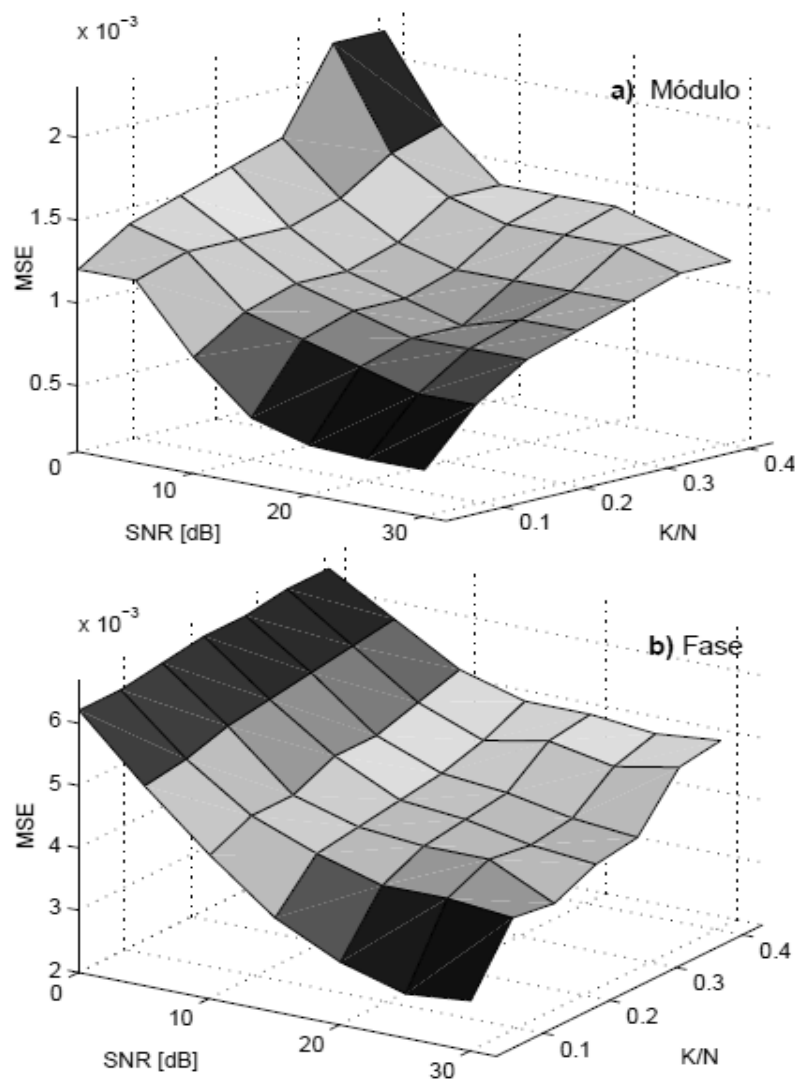

Fonte: do autor.

\section{Conclusões}

Neste trabalho foi aplicada uma metodologia de estimação de parâmetros de canal para sistemas DS/CDMA baseada na filtragem de partículas. O desempenho do estimador de coeficientes de canal M-BPF foi analisado sob diversos cenários de interesse prático: a) quando há apenas um usuário ativo no sistema; b) na situação de carregamento de sistema crescente; c) sob o efeito near-far e d) ampla faixa de SNR. Pôde-se observar que o desempenho do estimador M-BPF não é imune ao efeito da MAI. No entanto, mesmo sob a condição de elevado nível de MAI o patamar do erro quadrático médio atingido com o estimador PF proposto ainda é aceitável. Observou-se também que o estimador proporciona um erro menor para a estimativa em módulo do que para a estimativa da fase dos coeficientes de canal.

Com o aumento do índice de carregamento, temse um aumento da interferência de múltiplo acesso, ocasionando uma degradação do desempenho do estimador de coeficientes de canal proposto, tanto em módulo quanto em fase. Já em relação ao efeito near-far, o estimador também não é totalmente imune, tendo seu desempenho levemente afetado quando os níveis de potência dos sinais interferentes no receptor são elevados, da ordem de $+10 \mathrm{~dB}$ ou superior em relação à potência do usuário de interesse.

Em conclusão, mesmo não sendo totalmente imune à MAI, bem como quando existir disparidades de potência entre os usuários (efeito near-far crescente), o estimador M-BPF foi capaz de atingir bom desempenho em termos de MSE, sendo adequado para compor estruturas e sistemas de comunicação por espalhamento espectral DS/ CDMA, os quais exijam operação sob pequenos erros nas estimativas dos coeficientes de canal, para uma ampla gama de configurações de operação do sistema.

\section{Referências}

ABRÃO, T.; CIRIACO, F.; JESZENSKY, P. J. E. Detecção multiusuário utilizando algoritmos heurísticos evolucionários e de busca local. Semina - Ciências Exatas e Tecnológicas, Londrina, v. 32, n. 2, p. 235-244, 2004.

CANDY, J. V. Bootstrap Particle Filtering. IEEE Signal Processing Magazine, Santa Barbara, v. 24, n. 4, p. 73 $85,2007$. 
CIRIACO, F.; ABRÃO, T.; TOLEDO, A. F.; JESZENSKY, P. J. E. Jointly multi-user detection and channel estimation with genetic algorithm. Wireless Communication and Mobile Computing, London, v. 11, n. 6, p. 767-782, 2009.

DENT, P.; BOTTOMLEY, G. E.; CROFT, T. Modified Jakes fading model. Electronics Letters, New York, v. 29, p. 1162-1163, 1993.

DJURIC, P. M.; KOTECHA, J. H.; ZHANG, J.; HUANG, Y.; GHIRMAI, T.; BUGALLO, M. F.; MIGUEZ, J. Particle filtering. IEEE Signal Processing Magazine, New York, v. 20, n. 5, p. 19-38, 2003.

FU, X.; JIA, Y. An Improvement on Resampling Algorithm of Particle Filters. IEEE Transactions on Signal Processing, New York, v. 58, n. 10, p. 5414-5420, 2010.

GORDON, N.; SALMOND, D.; SMITH, A. F. M. Novel approach to nonlinear/nonGaussian Bayesian state estimation. IEE Proceedings Radar Signal Process, New York, v. 140, n. 2, p. 107-113, 1993.

GUIMARÃES, P. J. G. Estimativa de parâmetros de canal para DS-CDMA baseada na decomposição em subespaços. 2005. Dissertação (Mestrado em Engenharia) - Escola Politécnica da Universidade de São Paulo, Departamento de Telecomunicações e Controle, São Paulo.

HAN, Y.; LIANG, G. Adaptive multi-user detection based on robust particle filter in CDMA System. In: FIRST INTERNATIONAL WORKSHOP ON DATABASE TECHNOLOGY AND APPLICATIONS. Wuhan: First International Workshop on Database Technology and Applications, 2009. p. 390-393.

HAYKIN, S.; MOHER, M. Sistemas modernos de comunicações wireless. São Paulo: Bookman, 2008.

HU, B.; LAND, I.; PITON, R.; FLEURY, B. H. A bayesian framework for iterative channel estimation and multiuser decoding in coded DS-CDMA. In: IEEE Global Telecommunications Conference Electrical Engineering/Electronics, Computer, Communications and Information Technology Association, 50., 2007, Washington. Proceeding. Washington: IEEE, 2007.

JAKES, E. W. C. Microwave mobile communications. North Andover: John Wiley \& Sons, 1974.

LINZHOU, X.; XIN-HUA, Z.; SHAO-QING, Y.; WENTAO, F. An efficient particle filter with variable number of particles for bearings-only tracking. In: INTERNATIONAL CONFERENCE ON SIGNAL PROCESSING, 2010. p. 2395-2398. Disponível em: <http://ieeexplore.iee.org/ stamp/stamp.jsp?tp=\&arnumber $=5657082>$ Acesso em: 10 de setembro de 2012 às 18:00 horas.
RIBEIRO, R. O.; MUSSI, A. M.; ABRÃO, T. Bayesian Estimator by Particle Filter. In: MICROWAVE \& OPTOELECTRONICS CONFERENCE, 2011, Natal. Proceedings... Natal: IEEE, 2011.

TORRIERI, D. Principles of spread-spectrum communication systems. London: Springer Science, 2005.
Recebido em 16 Fevereiro 2012- Received on February 16, 2012.

Aceito em 28 Agosto, 2012 - Accepted on August 28, 2012. 
